\title{
Evaluating the histopathological and mechanical effects of a new forceps design: comparison of hemispheric bipolar forceps tip with usual bipolar tip on fresh cadaver cattle brain model
}

\author{
Adnan ALTUN ${ }^{1 *} *$ (i), Cengiz ÇOKLUK ${ }^{2}$ (i) \\ ${ }^{1}$ Department of Neurosurgery, Medical Faculty, Karatay University, Konya, Turkey \\ ${ }^{2}$ Department of Neurosurgery, Medical Faculty, Ondokuz Mayis University, Samsun, Turkey
}

\begin{abstract}
\begin{tabular}{lllll}
\hline Received: 05.03.2021 & $\bullet$ & Accepted/Published Online: 07.03.2021 & $\bullet$ & Final Version: 30.08 .2021
\end{tabular}
\section{Abstract}

Without electrocautery, many modern surgical interventions are practically impossible. In neurosurgery, bipolar cautery forceps has been evolved to not only be an auxiliary, but as a principal instrument wielded by the dominant hand of the surgeon to navigate through the most delicate tissue that there is. The purpose of this study is to introduce our original bipolar forceps designed exclusively for microneurosurgical interventions and compare its feasibility with a standard bipolar forceps tip. This study has been conducted on two fresh cadaveric cow brains under the operating microscope. The coagulative and ablative effects of the hemispheric bipolar forceps tip (HBFT) have been histologically compared with those of the standard bipolar forceps tip (SBFT). Likewise, their efficacies as a dissection instruments have been compared via performing dissections from the parietal surface down to the corpus callosum. HBFT proved less traumatic to the uninvolved brain tissue during dissection. Also, histological analyses have revealed that ablative effects of the HBFT are more confined to the bleeding point, more effectively sparing the uninvolved brain tissue. Results of this experimental study suggest that HBFT is a better instrument to be used in microneurosurgical interventions, along with other surgical disciplines where selective diathermy is critical.
\end{abstract}

Keywords: brain surgery, coagulation, hemispheric tip bipolar forceps, microsurgery

\section{Introduction}

Upon its introduction in 1940 by Greenwood, bipolar diathermy forceps revolutionized neurosurgical operations by not only providing a safe means for bleeding control, but also for navigating through the brain substance as well. Unlike unipolar cautery in which electricity courses from the entry point to the lead attached to a remote region of the patient; a lesser but more intense electric current passing between the tiny forceps tips carbonizes the vessels and stops the bleeding while minimally affecting the sensitive bystander neural tissue (1-5).

Microneurosurgery takes place in a tight spot. The luxury of changing instruments at whim is hardly afforded and the surgeons eventually came to rely on the tool in their dominant hand for practically everything, bleeding control, microdissection and even tumour ablation and removal. There are many types of bipolar forceps tips in use, including those used by the authors in their routine practice; yet none of them is specially designed for neurosurgery (1). It has been noted that although a neurosurgeon does much more than coagulation by these instruments, they are hardly any different than those used only for coagulation by other disciplines. Consequently, a bipolar forceps tip which is better suited to work as a multi tool inside arguably the most valuable tissue on earth has been designed.
A prototype with two subtypes has been constructed by a local medical instrument manufacturer and its efficacy has been compared with that of a SBFT on a cadaveric cattle brain model.

\section{Materials and methods}

No living creature was used in this study.

\subsection{Instrument design}

In the new design, features which better accommodate the conditions surrounding micro neurosurgery have been sought, including limited electrodessication, atraumatic dissection and efficient but selective tissue ablation. Unlike SBFT, the new design features a forceps the tips of which do not taper down to converge as a pointy end, but rather two concave hemispheres with bevelled edges which complement each other (model a). Another version (model b) includes not a smooth bevelled but toothed rim designated for handling denser masses (Fig. 1). HBFT's has been manufactured by a private medical instrument manufacturer company out of medical grade pure silver for the body and medical high quality silicone coating on the handles. And, Medtronic (USA) leads which fit standard Valley lab ${ }^{\mathrm{TM}}$ Medtronic (USA) electrocautery device sockets. The material properties of the HBFT are like those of the SBFT (Bahadır 
Electrosurgical Instruments, Turkey) used as the counterpart in this study.
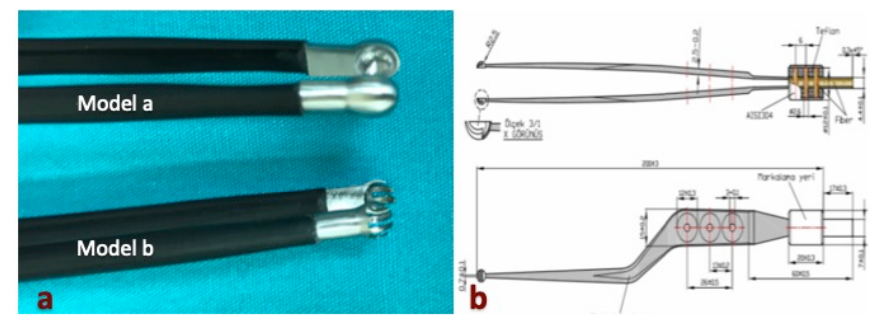

Fig. 1. Design of Hemispheric Bipolar Forceps Tips (HBFT). A: Smooth edged (top) and toothed (bottom) HBFT designs. B: Technical diagram

\subsection{Experimental design}

Three fresh cadaveric cattle brains have been used to assess the effectiveness of both types of HBFTs and electrical damage inflicted, dissection efficacy, and ablative efficacy have been compared with those yielded by SBFT. Parameters which are taken into consideration are as follows:

- With each instrument, cranial surfaces and pia maters of each brain have been cauterized five times with 15 watts power setting. Under the operating microscope, mechanical effects have been assessed.

- On each brain, cortical splits were done on both parietal lobes with a no. 15 scalpel blade and dissections were carried out with both instruments. During dissections, mechanical damage inflicted on the brain tissue (tears, punctures) was observed under the operating microscope.

- Within the subcortical parenchymal tissue, ten 15-watt coagulations were done on each brain and afterwards, cauterized specimens were harvested for histological analyses.

- A parenchymal tumour model was created using moderately boiled egg yolk in each brain's subcortical space. Egg yolk maintained the consistency of a mass while lacing the surrounding tissues, mimicking the surgical behaviour of a glial tumour. Afterwards they were cauterized away using both instruments. The effectiveness of tumour ablation and each tool's collateral damage was compared.

\section{Results}

When pia coagulation was mechanically evaluated, it has been observed that HBFT produces a homogenous, superficial coagulation zone while SBFT produces a deeper desiccation with a carbonized central puncture on all cauterizations (Fig. 2).

During cortical dissection, it has been observed that when the tips are closed the HBFT probes through the brain substance without allowing the tissue to get caught on any pointy end. When released to separate tissue, convex outer surfaces gently push the tissues apart without inflicting any tears. When the same feat is attempted by SBFT, it has been noted that despite best efforts, it is highly likely to inflict punctures and tears along the way. When closed, SBFT's needle like tip was highly likely to cut through tissue rather than dissecting it, and when separated the tips inflicted tears while pushing tissues apart, even with care was taken to avoid it (Fig. 3).
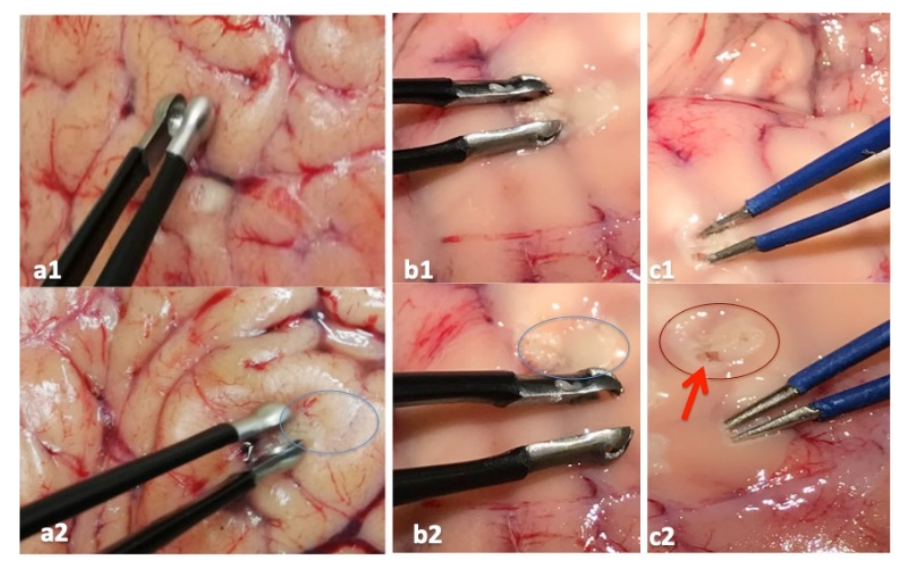

Fig. 2. a1-2: pia coagulation with HBFT (model a); b1-b2: pia coagulation with HBFT (model b); c:1-2 homogenous superficial coagulation zone without puncture with SBFT; red arrow: pucture on pia

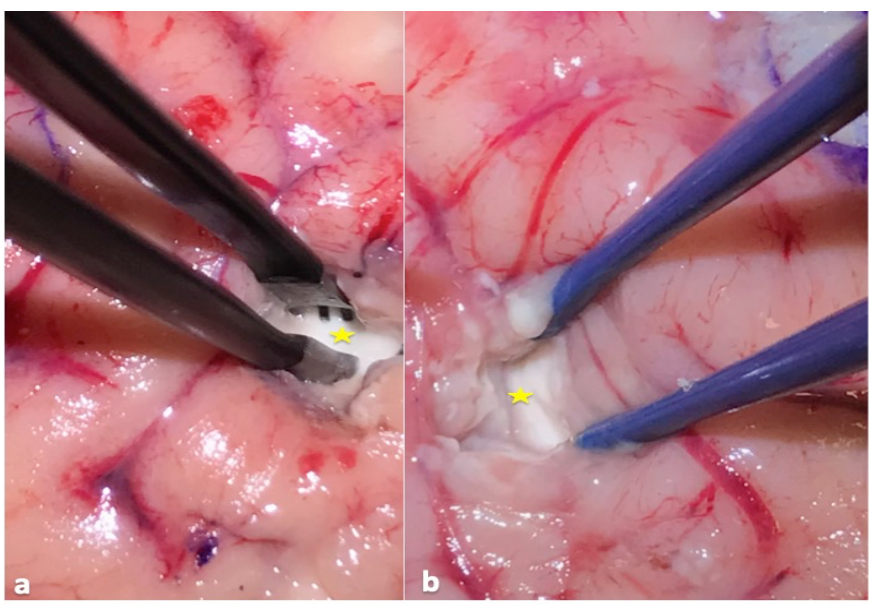

Fig. 3. a: cortical dissection with HBFT without inflicting any tears; b: cortical dissection with SBFT inflicting tears yellow star: corpus callosum

Both types of HBFT have proved as a highly effective tool for tumour ablation as well. Its scoop-like shape harvested the tumour while superficially cauterizing it away from the healthy tissue. The result was an evenly cauterized cavity, which is totally cleared of the tumour. This function could not be achieved by SBFT. Instead, it repeatedly chipped away on the tumour model without removing it (Fig. 4).

Histopathological evaluations further demonstrated the difference between the collateral damages associated with cautery use. The coagulation depth and the severity of desiccation were remarkably higher with the SBFT while the coagulation zone of the HBFT was subtler, more even and superficial (Fig. 5).

In tumour ablation, HBFT demonstrated a superficially desiccated parenchymal surface. The histological data pertaining to coagulation and tumour ablation of both tips has been summarized in Table 1. 


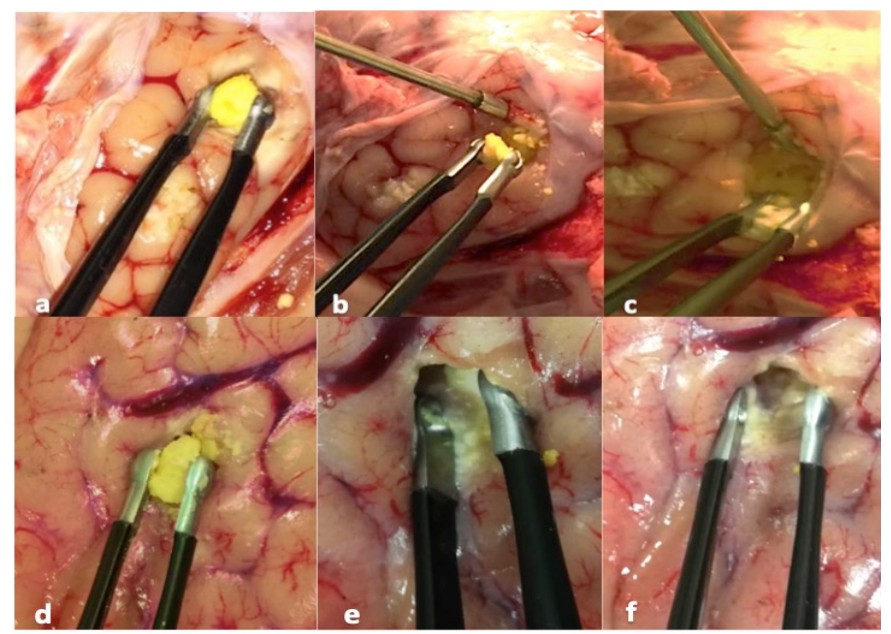

Fig. 4. A parenchymal tumour model was created using moderately boiled egg yolk. a-b-c tumour ablation with HBFT like tumour forceps (model a); d-e-f tumour ablation with HBFT like tumour forceps (model b)

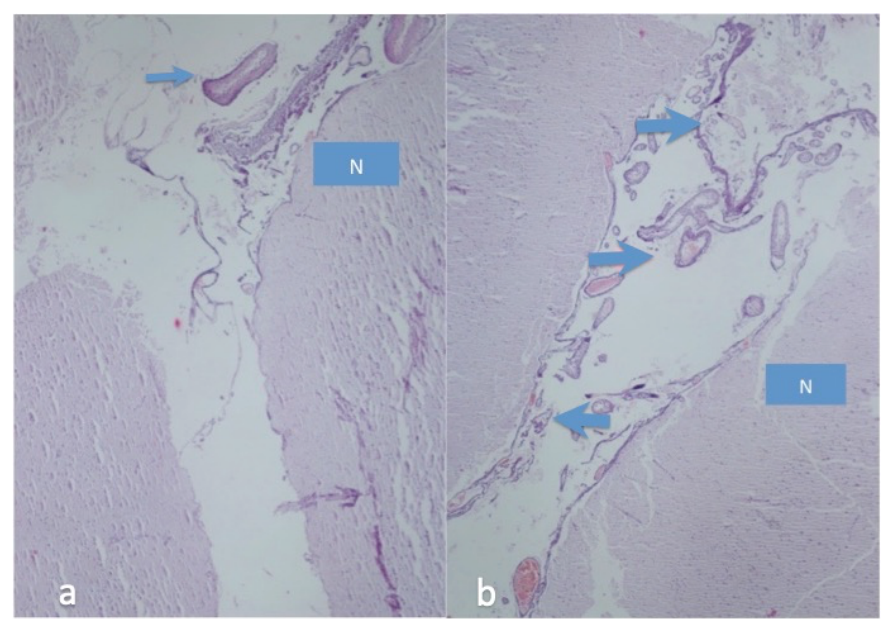

Fig. 5. Histopathological evaluations difference between the collateral damages associated with cautery use. a: The coagulation depth and the severity of desiccation were lower with the HBFT; blue arrow: shows cauterized veins only on the surface area; $\mathrm{N}$ : neuroglial tissue; b: The coagulation depth and the severity of desiccation were higher with the SBFT; blue arrows: show cauterized veins on the surface and deep area; N: neuroglial tissue

Table 1. The histological data pertaining to coagulation and tumour ablation of both SBFT and HBFT (HBFT model $a$ and $b$ )

\begin{tabular}{|l|l|l|l|}
$\begin{array}{l}\text { SBHT } \\
\text { Parenchymal } \\
\text { surface } \\
\text { coagulation } \\
\text { depth }\end{array}$ & $\begin{array}{l}\text { HBFT } \\
\text { Parenchymal } \\
\text { surface } \\
\text { coagulation } \\
\text { depth }\end{array}$ & $\begin{array}{l}\text { SBHT } \\
\text { Tumour } \\
\text { ablation } \\
\text { coagulation } \\
\text { depth }\end{array}$ & $\begin{array}{l}\text { HBFT } \\
\text { Tumour } \\
\text { ablation } \\
\text { coagulation } \\
\text { depth }\end{array}$ \\
\hline $5.3 \mathrm{~mm}$ & $3.1 \mathrm{~mm}$ & $6.2 \mathrm{~mm}$ & $4 \mathrm{~mm}$ \\
$5.3 \mathrm{~mm}$ & $3.2 \mathrm{~mm}$ & $6.2 \mathrm{~mm}$ & $4.1 \mathrm{~mm}$ \\
$6 \mathrm{~mm}$ & $3.2 \mathrm{~mm}$ & $6.4 \mathrm{~mm}$ & $4.2 \mathrm{~mm}$ \\
$6.4 \mathrm{~mm}$ & $3.4 \mathrm{~mm}$ & $6.4 \mathrm{~mm}$ & $4.2 \mathrm{~mm}$ \\
$7 \mathrm{~mm}$ & $4 \mathrm{~mm}$ & $6.9 \mathrm{~mm}$ & $4.4 \mathrm{~mm}$
\end{tabular}

\section{Discussion}

For many surgeons, bipolar forceps is an instrument that is used to zap bleeders, most of the time wielded by the assistant. For a neurosurgeon bipolar forceps is a multi-tool glued to his/her dominant hand until the job is done. It is effectiveness as a coagulation tool is of cardinal importance, as a haematoma as small as a few millimetres can have deleterious repercussions $(3,4)$. However, in neurosurgical practice it goes beyond that; since neurosurgeons can need the bipolar practically anytime, they tend to hold on to it throughout the surgery and use it for navigating through the brain substance. Therefore, a bipolar cautery forceps designed for such special circumstances must be more effective and less traumatic in dissecting brain tissue, minimally subject the surrounding tissues to electrical damage while effectively stopping the bleeding, and effectively bite away any tissue to be ablated while, again, minimally effecting the tissues other than those the surgeon wants to get rid of.

Since its use in neurosurgery has been introduced by Greenwood in 1940, bipolar cautery tips remained pretty much the same. Other than the popularization of bayonet design by Yaşargil, bipolar tips are hardly customized for carrying out these delicate tasks. Rather, neurosurgeons made do with the designs, which are available for general use.

As practitioners of neurosurgery, we believe that a bipolar forceps tip to be used exclusively in neurosurgery is much needed and developed a design which can overcome the shortcomings of the instruments readily available.

Classical pointed bipolar forceps tip is designed to cauterize small vessels and it effectively delivers. However, in neurosurgery not only tiny vessels, but also little sinusoid venules are also encountered, and their bleeders are very troublesome to control with the SBFT, if not at all impossible. HBFT has been shown to create a surface coagulation rather than a point coagulation, which is broader but more superficial, as would be desired in neurosurgical procedures.

We tried out the new design and compared it with a tip available for clinical use in a cadaveric model. While using a cadaveric, instead of live model can be regarded as a weakness of our study design, we believed that we needed a large brain to mimic the challenges encountered in actual brain surgery. It is our belief that researchers tend to use live specimens not necessarily because it is absolutely required but to increase the manuscript's chances of being accepted. Since we are using a clinical counterpart to assess efficacy, we concluded that sacrificing live subjects with a brain size comparable with humans was ethically and practically redundant.

Since neurosurgeons prefer to keep the bipolar readily available, using it for dissection of brain substance is commonplace. However, since it has not been manufactured to be used in this manner, it does not provide effective dissection and exposure with its slim body. Moreover, due to its sharp pointy end, it can easily inflict trauma to the surrounding tissues in all but steadiest of hands. HBFT has a smooth, blunt tip when it is closed, and it follows the natural cleavage of the brain substance. When the tip is opened, it gently pushes the tissues apart thanks to the wide and convex 
outer surface. In our experiment, it has been noted as a faster and safer means of cortical dissection.

When the tumour is accessed, the daunting task of fully removing it without bleeding out the patient stands. Therefore, the surgeon must switch between the tumour forceps and bipolar cautery tip, taking turns ablating the tumour and stopping the bleeding. This course of action is distracting, frustrating and time consuming; all of which are very undesirable during a brain surgery. In this situation HBFT also doubles as a tumour forceps. It cuts away the tumour, stops bleeding before it starts, control a bleeder if present, and leave a superficially desiccated cavity, ablating any remnant tumour substance along the way. In our experiment, both types of HBFT's were similarly atraumatic.

We believe that we came up with a design which can enhance the practice, speed, and success of micro neurosurgical procedures. The HBFT has been designed, as a response to the needs of neurosurgeons and requirements of neurosurgery and it is likely to be emulated by other surgical disciplines as well. The patent application is made and as of now pending.

\section{Conflict of interest}

The author(s) declared no potential conflicts of interest with respect to the research, authorship, and/or publication of this article.

\section{Funding}

The author(s) received no financial support for the research, authorship, and/or publication of this article.

\section{References}

1. Altunrende ME, Hamamcioglu MK, Hicdonmez T, Akcakaya MO, Birgili B, Cobanoglu S. Microsurgical training model for residents to approach to the orbit and the optic nerve in fresh cadaveric sheep cranium. J Neurosci Rural Pract. 2014; 5:151-4.

2. Belykh E, Byvaltsev V. Off-the-job microsurgical training on dry models: Siberian experience. World Neurosurg. 2014; 82: 20-4.

3. Spetzger U, von Schilling A, Brombach T, Winkler G: Training models for vascular microneurosurgery. Acta Neurochir. Suppl. 2011; 112:115-9.

4. Yadav YR, Parihar V, Ratre S, Kher Y, Iqbal M. Microneurosurgical skills training. J Neurol Surg A Cent Eur Neurosurg. 2016; 77:146-54.

5. Turan Suslu H, Ceylan D, Tatarlı N, Hicdonmez T, Seker A, Bayrı $\mathrm{Y}$, et al. Laboratory training in the retrosigmoid approach using cadaveric silicone injected cow brain. Br J Neurosurg. 2013; 27: 812-4. 